\title{
Association of a miR-502-Binding Site Single Nucleotide Polymorphism in the 3'-Untranslated Region of SET8 and the TP53 Codon 72 Polymorphism with Cervical Cancer in the Chinese Population
}

\author{
Shao-Di Yang ${ }^{1}$, Yan-Lin Cai ${ }^{2}$, Pei Jiang ${ }^{1 *}$, Wen Li ${ }^{1}$, Jian-Xin Tang ${ }^{1 *}$
}

\begin{abstract}
Objective: This study was conducted to identify whether polymorphic variants of set domain-containing protein 8 (SET8) and tumor protein p53 (TP53) codon 72, either independently or jointly, might be associated with increased risk for cervical cancer. Methods: We genotyped SET8 and TP53 codon 72 polymorphisms of peripheral blood DNA from 114 cervical cancer patients and 200 controls using the polymerase chain reactionrestriction fragment length polymorphism (PCR-RFLP) and direct DNA sequencing. Results: The frequency of SET8 CC (odds ratios $(\mathrm{OR})=\mathbf{2 . 7 1 7}, 95 \% \mathrm{CI}=\mathbf{1 . 4 3 6 - 5 . 1 4 1})$ or TP53 GG $(\mathrm{OR}=\mathbf{2 . 1 6 8 , 9 5 \%} \mathrm{CI}=\mathbf{1 . 1 4 9 - 4 . 0 8 9})$ genotype was associated with an increased risk of cervical cancer on comparison with the SET8 TT or TP53 CC genotypes, respectively. In additional, interaction between the SET8 and TP53 polymorphisms increased the risk of cervical cancer in a synergistic manner, with the OR being 9.913 (95\% CI=2.028-48.459) for subjects carrying both SET8 CC and TP53 GG genotypes. Conclusion: These data suggest that there are significant associations between the miR-502-binding site SNP in the 3'-UTR of SET8 and the TP53 codon 72 polymorphism with cervical cancer in Chinese, and there is a gene-gene interaction.
\end{abstract}

Keywords: microRNA - SET8 - TP53 - polymorphism - cervical cancer - synergism

Asian Pac J Cancer Prev, 15 (16), 6505-6510

\section{Introduction}

Cervical cancer is the second common gynecologic malignant tumor in the world, and it remains a leading cause of cancer-related death for women in developing countries (Akinyemiju, 2012; Ginsberg et al., 2012; Shuai et al., 2012; Gocze et al., 2013). Risk factors for developing cervical cancer are infection by the human papilloma virus (HPV), sexual behavior, diet, and genetic factors (Sitakan et al., 2013). Although the HPV infection is known to be a high-risk factor in cervical carcinogenesis, only a small proportion of such HPV-infected cases progress to cervical cancer. Thus, it indicates that genetic factors may be an important factor in the development of cervical cancer (Moore et al., 2012). Understanding of genetic variant that are associated with cervical cancer will contribute to identification of fundamental mechanism behind its formation, development and possible therapeutic target.

Set domain-containing protein 8 (SET8, also known as PR-SET7) is located on chromosme 12q24.31. It is a histone H4-Lys-20-specific methyltransferase that plays a significant role in cell-cycle-dependent transcriptional silencing and mitotic regulation in metazoans (Beck et al., 2012). Tumor protein p53 (TP53) gene is located on $17 \mathrm{p} 13.1$. The encoded TP53 protein responds to diverse cellular stresses to regulate expression of target genes, thereby inducing DNA repair, cell cycle arrest, and apoptosis (Kim et al., 2009). As a methyltransferase, SET8 methylates TP53 at Lys-382, which may affect the function of TP53. The deletion of SET8 augments the proapoptotic and checkpoint activation functions of TP53 (Tardat et al., 2007). Thus mutation in either SET8 or TP53 may lead to loss of homeostatic control during human carcinogenesis.

Single-nucleotide polymorphisms (SNPs) are the most frequent type of variation in the human genome, researchers have found SNPs that may help predict individual susceptibility to various cancer and response to certain drugs (Giacomini et al., 2007; Nicoloso et al., 2010). SNPs located in microRNA-binding site contribute to the disruption of microRNA-target interactions and regulate the expression of target gene. One of the 129 variants of the SET8 gene polymorphisms (rs16917496, $\mathrm{T} \rightarrow \mathrm{C}$ ) is located within the miR-502-binding site in the 3'-UTR. In recent years, reports have showed that

${ }^{1}$ Key Laboratory of Green Packaging and Application of Biological Nanotechnology, Hunan University of Technology, Zhuzhou, ${ }^{2}$ Department of Gynecology and Obstetrics, First Hospital Affiliated to Nanhua University, Hengyang, China *For correspondence: 15886311258@163.com 
the miR-502-binding site SNP in -the 3'-UTR of SET8 (rs16917496 C/T) was associated with the risk of breast cancer, hepatocellular carcinoma, epithelial ovarian cancer and so on (Song et al., 2009; Ding et al., 2012; Guo et al., 2012; Wang et al., 2012; Xu et al., 2013). However, there is no evidence showed a correlation between the miR-502-binding site SNP in the 3'-UTR of SET8 (rs16917496 C/T) and cervical cancer. The TP53 codon 72 polymorphism produces variant with $\mathrm{G} / \mathrm{C}$, and the variant lead to an amino acid change in the protein product (Arg $\rightarrow$ Pro). Some reports have showed the association between the TP53 codon 72 polymorphism and cervical cancer risk, but the results are controversial (Storey et al., 1998; Bhattacharya et al., 2005; Setteetham-Ishida et al., 2005; Clotti et al., 2006; Govan et al., 2007; Elkhair et al., 2010; Roh et al., 2010; Habbous et al., 2012; Singhal et al., 2013).

The present study is aimed at investigating the potential role of SET8 and TP53 interaction plays in the cell cycle control, DNA repair, cell apoptosis manner, and functional significant of variants, we conducted a casecontrol study to identify the relationship between SET8 or/and TP53 polymorphism (s), and cervical cancer risk among Chinese southern people.

\section{Materials and Methods}

\section{Study subjects and samples}

This study includes 114 newly diagnosed patients with cervical cancer and 200 cancers-free controls. Patients were recruited between January 2011 and June 2013 at The Central Hospital of Zhuzhou City (Zhuzhou, China). Final diagnoses of cases were confirmed by routine histopathological examination. The selection of criteria for the controls included no individual history of cancer and frequency, matched to case patients based on age. All subjects were unrelated ethnic Han Chinese. At recruitment, written informed consents about the study were obtained from all the patients and controls. Each participant was interviewed to collect information on demographic characteristics. The research protocol was approved by the Institutional Review Board of the hospital.

\section{SET8 polymorphism}

Polymorphism in SET8 was also determined by polymerase chain reaction-restriction fragment length polymorphism (PCR-RFLP) as previously described by Song et al (2009). PCR conditions were consisted of an initial denaturation at $95^{\circ} \mathrm{C}$ for 5 minutes, followed by 35 steps cycles of $95^{\circ} \mathrm{C}$ for 45 seconds, $66^{\circ} \mathrm{C}$ for 40 seconds, and $72^{\circ} \mathrm{C}$ for 30 seconds, and a final extension step of $72^{\circ} \mathrm{C}$ for 10 minutes. Ten microliters of PCR products were digested with SWAI restriction enzyme (New England Biolabs, Inc.) for overnight at $25^{\circ} \mathrm{C}$. When SWAI restriction site ( $\mathrm{T}$ allele) was present, the 308 base pairs (bp) fragment was digested into two small fragments (149 and $159 \mathrm{bp}$ ). The $\mathrm{C}$ allele was not cleaved by SWAI, having a single $308 \mathrm{bp}$ band. The CT heterozygote contained three bands, corresponding to 149, 159 and 308 bp (Figure 1A).

\section{TP53 codon 72 polymorphism}

TP53 codon 72 genotypes were detected using PCR-RFLP technique as previously described by Jiang et al (2010). The PCR profile consisted of an initial denaturation at $94^{\circ} \mathrm{C}$ for 2 minutes, followed by 35 step cycles of $94^{\circ} \mathrm{C}$ for 30 seconds, $60^{\circ} \mathrm{C}$ for 30 seconds, and $72^{\circ} \mathrm{C}$ for seconds, and a final extension step of $72^{\circ} \mathrm{C}$ for 10 minutes. Digestion was done in 20 microliters reaction volume using BstUI restriction enzyme (New England Biolabs, Inc.) for 2 hours at $60^{\circ} \mathrm{C}$ according to the instructions of the manufacturer. The fragments were visualized in $2.5 \%$ agarose gel electrophoresis. $\mathrm{C}$ alleles produced a single band of $279 \mathrm{bp}$, which was caused by lack of BstUI restriction site; G alleles were digested to produce two bands of 119 and $160 \mathrm{bp}$. Thus, the CG heterozygote produced three bands, corresponding to 119 , 160, 279 bp (Figure 1B).

The genotypes within $10 \%$ of each sample group were confirmed by sequencing the PCR products using an automated sequencing system (ABI Applied Biosystems, Foster City, USA). To test the reliability of the assay, 50 randomly selected samples were re-tested with identical results.

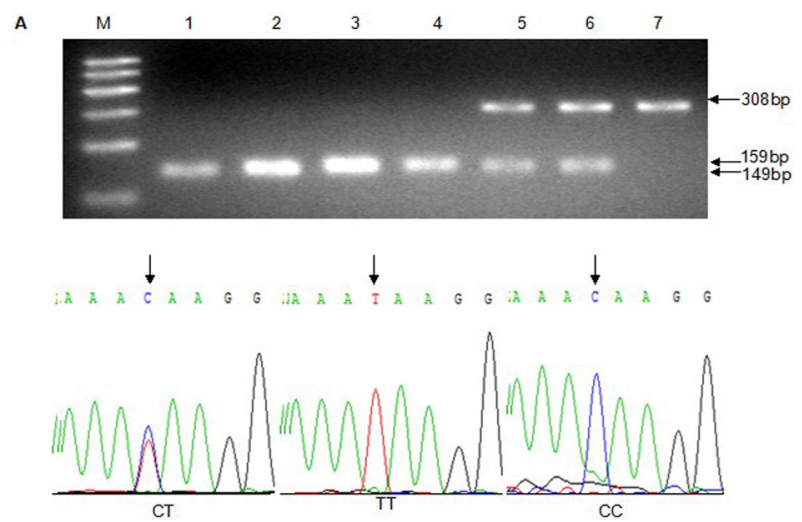

B
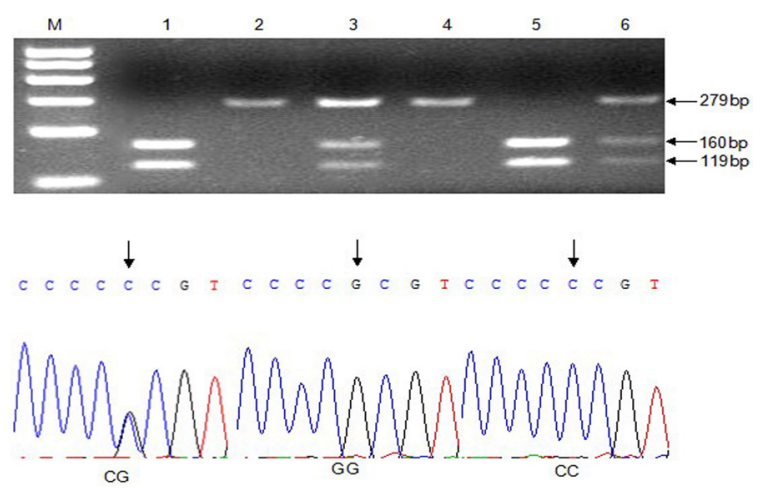

Figure 2. The PCR-RFLP Analyses and Directsequence Result of SET8 and TP53 Codon 72 Polymorphisms. A: SET8 polymorphisms. Lane 1, 2, 3 and 4, TT homozygous genotypes (159 and $149 \mathrm{bp}$ ); lanes 5, and 6, CT heterozygous genotype (308, 159 and $149 \mathrm{bp}$ ); lanes 7, CC homozygous genotype (308 bp). B: TP53 codon 72 polymorphisms. Lanes 1 , and 5, GG homozygous genotypes (160, and $119 \mathrm{bp}$ ); lanes 2, and 4, CC homozygous genotypes (279 bp); lane 3 and 6, CG heterozygous genotype $(279,160$ and $119 \mathrm{bp})$ 


\section{Statistical analysis}

We used the $\chi^{2}$-test to evaluate the differences in age, cigarette smoking, alcohol consumption and family history between cases and controls. The association between the polymorphisms and cervical cancer risk were determined by odds ratios (ORs) and $95 \%$ confidence intervals (CIs) by unconditional logistic regression models. P-values less than 0.05 were considered statistically significant. The Statistical Package for the Social Sciences 13.0 (Statistical Product and Service Solutions Company, Chicago) was used for all statistical analyses.

\section{Results}

\section{Characteristics of study subjects}

The frequency distribution of selected characteristics of the cases and controls is presented in Table 1. The frequency distribution between age, alcohol consumption and family history had no significant difference in cases and controls. However, the smokers' frequency among cases and controls was $17.5 \%$ and $6.5 \%(p=0.002)$, which indicated smoking was a high risk factor for cervical cancer.

Table 1. Demographic Characteristic of Cervical Cancer Cases and Controls

\begin{tabular}{lcccc}
\hline Characteristics & $\begin{array}{c}\text { Cases } \\
(\%)\end{array}$ & $\begin{array}{c}\text { Controls } \\
(\%)\end{array}$ & $\begin{array}{l}P \text {-value } \\
\chi^{2} \text {-value }\end{array}$ \\
\hline Total & 114 & 200 & & \\
Age & & & 0.258 & 1.279 \\
$\leq 45$ & $54(47.4)$ & $108(54.0)$ & & \\
$>45$ & $60(52.6)$ & $92(46.0)$ & & \\
Cigarette smoking & & & 0.002 & 9.416 \\
No & $94(82.5)$ & $187(93.5)$ & & \\
Yes & $20(17.5)$ & $13(6.5)$ & & \\
Alcohol consumption & $91(79.8)$ & $164(82.0)$ & & \\
No & $23(20.2)$ & $36(18.0)$ & & \\
Yes & & & 0.225 \\
Family history & $10(8.8)$ & $10(5.0)$ & & \\
Yes & $104(91.2)$ & $190(95.0)$ & & \\
No & & & \\
Histology & $9(8.0)$ & & \\
Adenocarcinoma $103(90.4)$ & & & \\
Squamous cell & $9(1.6)$ & & \\
Others & & & \\
\hline
\end{tabular}

Two-sided $\chi^{2}$-text

\section{Distribution of SET8 and TP53 polymorphisms}

The allele and genotype frequencies of SET8 and TP53 polymorphisms are showed in Table 2 . The observed genotype frequencies of SET8 and TP53 polymorphisms in controls did not deviate significantly from those expected from the Hardy-Weinberg equilibrium. The frequencies of SET8 $\mathrm{C}$ allele, and TP53 $\mathrm{G}$ allele were $43.0 \%$, and $62.3 \%$ among cases, and $28.7 \%$ and $51.5 \%$ among controls, respectively. The frequencies of the SET8 TT, CT, and CC genotypes in patients were significantly different from those in controls, with the CC genotype being more frequent among cases compared with controls (24.6\% vs $13.0 \% ; p<0.05)$. Similarly, The frequencies of the TP53 CC, CG, and GG genotypes in patients were significantly different from those in controls, with the GG variant being more frequent among cases compared with controls $(42.1 \%$ vs $31.0 \%$; $p<0.05)$.

The association between SET8 and TP53 polymorphisms and cervical cancer risk

An unconditional logistic regression model was used to estimate the association between genotypes and the risk of cervical cancer in Table 2. The SET8 CC genotype was associated with an increased risk for the development of cervical cancer $(\mathrm{OR}=2.717,95 \% \mathrm{CI}=1.436-5.141)$, compared with the TT genotype. Likewise, the TP53 GG genotype was also associated with an increased risk of developing cervical cancer $(\mathrm{OR}=2.168,95 \% \mathrm{CI}=1.149$ 4.089), compared with the CC genotype. However, the heterozygous genotypes for both polymorphisms (SET8 CT or TP53 CG) were not associated with the risk. We combined the SET8 TT and CT or TP53 CC and CG into one group for subsequent analysis. We examined whether there was a statistical joint effect between the SET8 and TP53 polymorphisms in Table 3. The observation showed that patients carrying the SET8 CC genotype were more likely to carry the TP53 GG genotype than the controls ( $7.0 \%$ vs $1.0 \%, p<0.05)$. Moreover, the OR significantly increased to $9.913(95 \% \mathrm{CI}=2.028-48.459)$ among subjects carrying both SET8 CC and TP53 GG genotypes. These results clearly indicated a significant interaction between the SET8 CC and TP53 GG genotype on the increased risk of developing cervical cancer according to the statistical model.

Table 2. Genotype and Allele Frequencies of SET8 and TP53 Among Cases and Controls and their Association with The Risk of Cervical Cancer

\begin{tabular}{|c|c|c|c|c|c|c|}
\hline \multirow{2}{*}{$\begin{array}{l}\text { Genotype } \\
\text { SET8 (T/C) }\end{array}$} & \multicolumn{2}{|c|}{$\begin{array}{l}\text { Cases } \\
(n=114)\end{array}$} & \multicolumn{2}{|c|}{$\begin{array}{l}\text { Control } \\
(n=200)\end{array}$} & \multirow[t]{2}{*}{$P$-value } & \multirow[t]{2}{*}{$\mathrm{OR}^{\mathrm{a}}(95 \% \mathrm{CI})$} \\
\hline & & & & & & \\
\hline TT & 44 & 38.6 & 111 & 55.5 & & 1 (reference) \\
\hline $\mathrm{CT}$ & 42 & 36.8 & 63 & 31.5 & 0.051 & $1.682(0.996-2.840)$ \\
\hline $\mathrm{CC}$ & 28 & 24.6 & 26 & 13 & 0.002 & $2.717(1.436-5.141)^{*}$ \\
\hline $\mathrm{T}$ allele frequency & 130 & 57 & 285 & 71.3 & & 1 (reference) \\
\hline $\mathrm{C}$ allele frequency & 98 & 43 & 115 & 28.7 & 0 & $1.868(1.330-2.625)^{*}$ \\
\hline \multicolumn{7}{|l|}{ TP53 codon72 $(\mathrm{C} / \mathrm{G})$} \\
\hline $\mathrm{CC}$ & 20 & 17.5 & 56 & 28 & & 1 (reference) \\
\hline $\mathrm{CG}$ & 46 & 40.4 & 82 & 41 & 0.156 & $1.571(0.840-2.935)$ \\
\hline GG & 48 & 42.1 & 62 & 31 & 0.016 & $2.168(1.149-4.089) *$ \\
\hline $\mathrm{C}$ allele frequency & 86 & 37.7 & 194 & 48.5 & & 1 (reference) \\
\hline $\mathrm{G}$ allele frequency & 142 & 62.3 & 206 & 51.5 & 0.009 & $1.555(1.116-2.167)^{*}$ \\
\hline
\end{tabular}

${ }^{\mathrm{a} O R s}$ were adjusted for age, sex, cigarette smoking, alcohol consumption and family history; ${ }^{*} p<0.05$ 
Table 3. Risk of Cervical Cancer Associated with SET8 Genotypes by TP53 Genotypes

\begin{tabular}{|c|c|c|c|c|c|c|c|}
\hline \multicolumn{2}{|c|}{ Genotype } & \multicolumn{2}{|c|}{ Cases $(n=114)$} & \multicolumn{2}{|c|}{ Controls $(n=200)$} & \multirow[t]{2}{*}{$P$-value } & \multirow[t]{2}{*}{$\mathrm{OR}^{\mathrm{a}}(95 \% \mathrm{CI})$} \\
\hline SET8 & TP53 codon 72 & & & & & & \\
\hline $\mathrm{TT}+\mathrm{CT}$ & $\mathrm{CC}+\mathrm{CG}$ & 46 & 40.4 & 114 & 57 & & 1 (reference) \\
\hline $\mathrm{TT}+\mathrm{CT}$ & GG & 40 & 35.1 & 60 & 30 & 0.061 & $1.652(0.976-2.797)$ \\
\hline $\mathrm{CC}$ & $\mathrm{CC}+\mathrm{CG}$ & 20 & 17.5 & 24 & 12 & 0.036 & $2.065(1.041-4.097) *$ \\
\hline $\mathrm{CC}$ & GG & 8 & 7 & 2 & 1 & 0.001 & $9.913(2.028-48.459) *$ \\
\hline
\end{tabular}

${ }^{a}$ ORs were adjusted for age, sex, cigarette smoking, alcohol consumption and family history; ${ }^{*} p<0.05$

\section{Discussion}

To our best knowledge, this is the first study demonstrating the effects of the association between the miR-502-binding site SNP (rs16917496) in the 3'-UTR of SET8 and the TP53 codon 72 polymorphisms and the risk of cervical cancer. In a cervical cancer case-control analysis, we found that both SET8 rs16917496 and TP53 codon 72 polymorphisms were associated with an increased risk for development of cervical cancer. Moreover, an increased multiplicative interaction between SET8 CC and TP53 codon 72 GG genotypes was also detected.

SET8 is a member of the SET domain-containing methyltransferase family especially targeting H4K20 for monomethylation and exerts diverse functions in transcription regulation (Congdon et al., 2010; Li et al., 2011), cell-cycle progression (Jogensen et al., 2007; Abbas et al., 2010; Centore et al., 2010) and genome stability (Houston et al., 2008; Oda et al., 2009). It has been reported that a SNP (rs16917496) in the miR502-binding site modulates SET8 gene expression, and some researches have studied the relationship between the miR-502-binding site SNP of SET8 gene and the susceptibility of different tumors. The homozygote CC genotype of SET8 gene rs16917496 has been found to decrease the risk of epithelial ovarian cancer in Chinese patients (Wang et al., 2012). Ding et al (2012) found that the SET8 CC+CT genotype was independently associated with longer survival in small cell lung cancer patients by multivariate analysis. On the contrary, Song et al (2009) found that the CC genotype of SET8 gene rs 16917496 is significantly increased the risk of the breast cancer in premenopausal women. However, there was no significant association between the SET8 CC and TT genotypes and postmenopausal women with breast cancer. Our study was consistent with Song et al (2009) that the SET8 CC genotype was associated with an increased risk for cervical cancer when compared with the SET8 TT genotype.

TP53 encodes a tumor suppressor protein, which plays multiple roles in apoptosis, cell-cycle control, and DNA repair (Hollstein et al., 2010). One of the most well studied TP53 gene polymorphism is located at codon 72 in exon 4, leading to arginine-to-proline substitution. Mutations in TP53 codon 72 are associated with a variety of human cancers, including cervical cancer. Since Storey et al (1998) investigated the arginine-encoding allele represents a significant risk factor in the development of HPV-associated cancers, TP53 codon 72 has received considerable attention over the last few years, but with the inconsistent results. Singhal et al (2013) found that GG genotype in TP53 codon 72 was associated with a higher risk in cervical cancer. Clotti et al (2006) studied Italy women with cervical cancer, finding that GG homozygous genotypes are at increased risk for cervical cancer. Contrary to these findings, no significant association between TP53 codon 72 and cervical cancer was observed in South Africa, Moroccan, northeastern Thailand, and East Asian population (Setteetham-Ishida et al., 2005; Govan et al., 2007; Elkhair et al., 2010; Habbous et al., 2012). Furthermore, Roh et al (2010) studied the TP53 codon 72 containing the $\mathrm{C}$ allele was significantly increased the risk of cervical adenocarcinoma in Korean women. In addition, Bhattacharya et al (2005) provided evidence that the $\mathrm{CC}$ genotype was a risk factor in cervical cancer among Indian women. Zhou et al (2012) demonstrated that the Pro/Pro (CC) genotype and Pro (C) carrier showed significant associations with increased the risk of cervical cancer among Indian population, but not among Chinese, Japanese and Korean populations by current meta-analysis. Our finding confirmed the initial result of Storey et al (1998), as well as the data of those that detected a higher prevalence of homozygosity for GG genotype in patients with cervical cancer.

SET8 has a well-defined function in the TP53 pathway by monomethylating TP53 at K382. SET8 methylates TP53 and thus regulates cell proliferation and genome stability (Shi et al., 2007). Deletion of SET8 augments the pro-apoptotic and checkpoint activation functions of TP53, and the expression of SET8 is down-regulated in DNA damage. Song et al (2009) reported a genotype dose-dependent pattern on the SET8 and TP53 codon 72 polymorphisms at the early age of breast cancer. It has been evaluated that the miR-502-binding site SNP (rs16917496) of the SET8 gene modulates SET8 expression, thus influencing its methylation of TP53 and contributing to the TP53 pathway-mediated breast cancer development. Our results also provided evidence that there is a gene-gene interaction between SET8 and TP53 polymorphsims in the risk of cervical cancer. However, a limitation of the present study is the small number of patients with each category of gene type, which may affect the estimates of ORs for genotypes. Further studies with large sample sizes are needed to confirm our finding. Furthermore, to better characterize the effect of gene on cervical cancer risk, it is necessary to incorporate other factors (HPV, sexual behavior et al) into the study.

In summary, our study showed a significant association between the polymorphism (rs16917496) of the miR-502binding site in the 3'-UTR of SET8 or/and TP53 codon 72 polymorphism and the risk of developing cervical cancer. The association of SET8 and TP53 polymorphisms with the risk of cervical cancer displayed a multiplicative genegene interaction. Further studies are warranted to elucidate 
the role of genetic polymorphisms in microRNA-related genes in cervical carcinogenesis.

\section{Acknowledgements}

This research is supported by the National Natural Science Foundation of China (No. 61171061), the Technology Planning Project of Hunan Province (No.2014SK2019), and the Natural Science Foundation of Hunan Province, China (No. 14JJ2149, No. 12JJ4032, and No. 12JJ4082).

\section{References}

Abbas T, Shibata E, Park J, et al (2010). CRL4 (Cdt2) regulates cell proliferation and histone gene expression by targeting PR-Set7/Set8 for degradation. Mol Cell, 40, 9-21.

Akinyemiju TF (2012). Socio-economic and health access determinants of breast and cervical cancer screening in low-income countries: analysis of the world health survey. PLoS One, 7, e48834.

Beck DB, Oda H, Shen SS, Reinberg D (2012). PR-Set7 and H4K20me1: at the crossroads of genome integrity, cell cycle, chromosome condensation and transcription. Genes \& Dev, 26, 325-37.

Bhattacharya P, Sengupta S (2005). Lack of evidence that proline homozygosity at codon 72 of p53 and rare arginine allele at codon 31 of $\mathrm{p} 21$, jointly mediate cervical cancer susceptibility among Indian women. Gynecol Oncol, 99, 176-82.

Centore RC, Havens CG, Manning AL, et al (2010). CRL4 (Cdt2)-mediated destruction of the histone methyltransferase Set8 prevents premature chromatin compaction in $\mathrm{S}$ phase. Mol Cell, 40, 22-33.

Clotti M, Colrtti A, Giuliani L, et al (2006). The p53 codon 72 arg/arg homozygous women in central Italy are at increased risl for HPV infections. Anticancer Res, 26, 3745-8.

Congdon LM, Houstion SI, Veerappan CS, et al (2010). PRSet7-mediated monomethylation of histone H4 lysine 20 at specific genomic regions induces transcriptional repression. J Cell Biochem, 110, 609-19.

Ding C, Li R, Peng J, Li S, Guo Z (2012). A polymorphism at the miR-502 binding site in the 3'untranslated region of the SET8 gene is associated with the outcome of small-cell lung cancer. Exp Ther Med, 3, 689-92.

Elkhair MM, Ennaji MM, El Kebbaj R, et al (2010). P53 codon 72 polymorphism and risk of cervical carcinoma in Moroccan women. Med Oncol, 27, 861-6.

Giacomini KM, Brett CM, Aitman RB, et al (2007). The pharmacogenetics research network: from SNP discovery to clinical drug response. Clinical Pharmacology \& Therapeutics, 81, 328-45.

Ginsberg GM, Lauer JA, Zelle S, Baeten S, Baltussen R (2012). Cost effectiveness of strategies to combat breast, cervical, and colorectal cancer in sub-Saharan Africa and South East Asia: mathematical modelling study. BMJ, 344, e614.

Gocze K, Gombos K, Juhasz K, et al (2013). Unique microRNA expression profiles in cervical cancer. Anticancer Res, 33, 2561-7.

Govan VA, Loubser S, Saleh D, Hoffman M, Williamson AL (2007). No relationship observed between human p53 codon-72 genotype and HPV-associated cervical cancer in a population group with a low arginine- 72 allele frequency. Int J Immunogenet, 34, 213-7.

Guo Z, Wu C, Wang X, et al (2012). A polymorphism at the miR-
502 binding site in the 3'-untranslated region of the histone methyltransferase SET8 is associated with hepatocellular carcinoma outcome. Int J Cancer, 131, 1318-22.

Habbous S, Pang V, Eng L, et al (2012). P53 Arg72Pro polymorphism, HPV statues and initiation, progression, and development of cervical cancer: a systematic review and meta-analysis. Clin Cancer Res, 18, 6407-15.

Hollstein M, Hainaut P (2010). Massively regulated genes: the example of TP53. J Pathology, 220, 164-73.

Houston SI, Mcmanus KJ, Adams MM, et al (2008). Catalytic function of the PR-Set7 histone H4 lysine 20 monomethyltransferase is essential for mitotic entry and genomic stability. J Biol Chem, 283, 19478-88.

Jiang P, Liu J, Zeng X, Li W, Tang J (2010). Association of TP53 codon 72 polymorphism with cervical cancer risk in Chinese women. Cancer Genetics and Cytogenetics, 197, 174-8.

Jøgensen S, Elvers I, Trelle MB, et al (2007). The histone methyltransferase SET8 is required for S-phase Progression. J Cell Biol, 179, 1337-45.

Kim T, Lee SY, Rho JH, et al (2009). Mutant p53 (G199V) gains antiapoptotic function through signal transducer and activator of transcription 3 in anaplastic thyroid cancer cells. Mol Cancer Res, 7, 1645-54.

Li Z, Nie F, Wang S, Li L (2011). Histone H4 Lys 20 monomethylation by histone methylase SET8 mediates Wnt target gene activation. Proc Natl Acad Sci USA, 108, 3116-23.

Moore EE, Wark JD, Hopper JL, et al (2012). The role of genetic and environmental factors on risk of cervical cancer: a review of classical twin studies. Twin Res Hum Genet, 15, 79-86.

Nicoloso MS, Sun H, Spizzo R, et al (2010). Single-nucleotide polymorphisms inside microRNA target sites influence tumor susceptibility. Cancer Res, 70, 2789-98.

Oda H, Okamoto I, Murphy N, et al (2009). Monomethylation of histone H4-lysine 20 is involved in chromosome structure and stability and is essential for mouse development. $\mathrm{Mol}$ Cell Biol, 29, 2278-95.

Roh JW, Kim BK, Lee CH, et al (2010). P53 codon 72 and p21 codon 31 polymorphisms and susceptibility to cervical adenocarcinoma in Korean women. Oncol Res, 18, 453-9.

Setteetham-Ishida W, Kanjanavirojkul N, Kularbkaew C, Ishida $\mathrm{T}$ (2005). Human papillomavirus genotypes and the p53 codon 72 polymorphism in cervical cancer of Northeastern Thailand. Microbiol Immunol, 49, 417-21.

Shi X, Kachirskaia I, Yamaguchi H, et al (2007). Modulation of p53 function by SET8-mediated methylation at lysine 382 . Mol Cell, 27, 636-46.

Shuai HL, Luo X, Yan RL, et al (2012). XRCC1 polymorphisms are associated with cervical cancer risk and response to chemotherapy: a systematic review and meta-analysis. Asian Pac J Cancer Prev, 13, 6423-7.

Singhal P, Hussain S, Thakur N, et al (2013). Association of MDM2 and 553 polymorphisms with the advancement of cervical carcinoma. DNA Cell Biol, 32, 19-27.

Sitakan N, Wannapa SI, Chamsai P, et al (2013). Human papillomavirus genotypes and cervical cancer in Northeast Thailand. APJCP, 14, 6961-4.

Song F, Zheng H, Liu B, et al (2009). An miR-502-binding site single-nucleotide polymorphism in the 3'-Untranslated region of the SET8 gene is associated with early age of breast cancer onset. Clin Cancer Res, 15, 6292-300.

Storey A, Thomas M, Kalita A, et al (1998). Role od a p53 polymorphism in the development of human papillomavirusassciated cancer. Nature, 393, 229-34.

Tardat M, Murr R, Herceg Z, Sardet C, Julien E (2007). PR-Set7dependent lysine methylation ensures genome replication and stability through S phase. J Cell Biol, 179, 1413-26. 
Wang C, Guo Z, Li Y, Kang S (2012). A polymorphism at the miR-502 binding site in the 3' untranslated region of the SET8 gene is associated with the risk of epithelial ovarian cancer. Cancer Genet, 205, 373-6.

Xu J, Yin Z, Gao W, et al (2013). Genetic variation in a microRNA-502 minding site in SET8 gene confers clinical outcome of non-small cell lung cancer in a Chinese population. PLoS ONE, $\mathbf{8}, \mathrm{e} 77024$.

Zhou X, Gu Y,Zhang SL (2012). Association between p53 codon 72 polymorphism and cervical cancer risk among Asians: a huge review and meta-analysis. Asian Pac J Cancer Prev, 13, 4909-14. 